\title{
Interpreting LHC Higgs results from natural new physics perspective
}

\author{
Dean Carmi, ${ }^{a}$ Adam Falkowski, ${ }^{b}$ Eric Kuflik $^{a}$ and Tomer Volansky ${ }^{a}$ \\ ${ }^{a}$ Raymond and Beverly Sackler School of Physics and Astronomy, Tel-Aviv University, \\ Tel-Aviv 69978, Israel \\ ${ }^{b}$ Laboratoire de Physique Théorique d'Orsay, UMR8627-CNRS, Université Paris-Sud, \\ Orsay, France \\ E-mail: carmidea@post.tau.ac.il, afalkows017@gmail.com, \\ ekuflik@gmail.com, tomerv@post.tau.ac.il
}

ABSTRACT: We analyze the 2011 LHC and Tevatron Higgs data in the context of simplified new physics models addressing the naturalness problem. These models are expected to contain new particles with sizable couplings to the Higgs boson, which can easily modify the Higgs production cross sections and branching fractions. We focus on searches in the $h \rightarrow Z Z^{*} \rightarrow 4 l, h \rightarrow W W^{*} \rightarrow l \nu l \nu, h \rightarrow \gamma \gamma, h j j \rightarrow \gamma \gamma j j$ and $h V \rightarrow b \bar{b} V$ channels. Combining the available ATLAS, CMS, and Tevatron data in these channels, we derive constraints on an effective low-energy theory of the Higgs boson. We then map several simplified scenarios to the effective theory, capturing numerous natural new physics models such as supersymmetry and Little Higgs, and extract the constraints on the corresponding parameter space. We show that simple models where one fermionic or one scalar partner is responsible for stabilizing the Higgs potential are already constrained in a non-trivial way by LHC and Tevatron Higgs data.

Keywords: Higgs Physics, Beyond Standard Model

ARXIV EPRINT: 1202.3144 


\section{Contents}

1 Introduction 1

$\begin{array}{lll}2 & \text { Formalism } & 3\end{array}$

3 Constraints from the LHC and Tevatron 5

4 Models with scalar top partners $\quad 8$

4.1 One scalar 8

4.2 Two scalars (MSSM) 8

5 Models with a fermionic top partner $\quad 10$

$\begin{array}{lll}5.1 \text { No mixing } & 12\end{array}$

$\begin{array}{ll}5.2 \text { Universal suppression } & 12\end{array}$

$\begin{array}{lll}5.3 & \text { Non-universal suppression } & 13\end{array}$

6 Multi-Higgs models $\quad \mathbf{1 5}$

$\begin{array}{lll}6.1 & \text { Doublet }+ \text { singlet } & 15\end{array}$

$\begin{array}{ll}6.2 \text { Two Higgs doublets } & 15\end{array}$

$\begin{array}{lll}7 & \text { Conclusions } & 17\end{array}$

\section{Introduction}

Discovering the Higgs boson and measuring its mass and branching ratios is one of the key objectives of the LHC. Within the Standard Model (SM), the coupling to the Higgs boson is completely fixed by the particle mass. This is no longer the case in many scenarios beyond the SM, where the Higgs couplings to the SM gauge bosons and fermions may display sizable departures from the SM predictions. Indeed, precision studies of the Higgs couplings may be the shortest route to new physics.

Interestingly, from this point of view, a Higgs boson in the range $115-130 \mathrm{GeV}$ is particularly well suited as a new physics probe. One reason is that several different Higgs decay channels, in particular the $\gamma \gamma, Z Z^{*}, W W^{*}$, and $b \bar{b}$ channels, can be realistically accessed by experiment. The first of these arises in the SM at one loop and, consequently, physics beyond the SM may easily modify its rate. This is especially true in models addressing the naturalness problem of electroweak symmetry breaking, which necessarily contain new charged particles with significant couplings to the Higgs boson. Well-known examples where this is the case include supersymmetric or composite Higgs models. Furthermore, the tree-level Higgs coupling to $W W, Z Z$, and $b \bar{b}$ is often modified as well, as is the case in composite or multi-Higgs models. Similar comments apply to the Higgs production 
rate: the dominant production mode via gluon fusion is a one loop process in the SM and is therefore particularly sensitive to new physics containing, as in typical natural models, light new colored states coupled to the Higgs. Subleading production modes, such as vector boson fusion (VBF) and associate production, may also be affected.

While several Higgs production and decay modes may change in the presence of new particles, the correlated change in different channel may crucially depend on the new physics scenario. Consequently, a joint analysis of distinct independent channels may either allow to place interesting bounds on new physics scenarios or otherwise provide a way to discover new physics and pinpoint its identity. The goal of this paper is to demonstrate the above understanding in light of the new Higgs measurements at the LHC and Tevatron, and place constraints on new physics models which solve the fine-tuning problem.

Recently, ATLAS, CMS, have reported the results of Higgs searches based on $5 \mathrm{fb}^{-1}$ of data in several channels [1-9, 9-13, 15], and the Tevatron reported on the $b \bar{b}$ channel [14]. The results, albeit inconclusive, suggest the existence of a Higgs boson with mass near $125 \mathrm{GeV}$. It is therefore natural to try and answer the following question: Assuming a Higgs boson with the mass $120 \mathrm{GeV} \leq m_{h} \leq 130 \mathrm{GeV}$, what are the implications of these results for natural models beyond the SM? Below we pursue this question.

We combine the latest ATLAS, CMS, and Tevatron Higgs results. Our focus is to interpret the results in terms of simple (sometimes simplified) models that address the finetuning problem in the sense of providing a new contribution to the Higgs mass that cancels the quadratically divergent contribution of the SM top quark. To do so, we first consider the Higgs effective action at low energy and derive the constraints on its couplings. We then map various theories onto the effective action to extract their bounds. A number of partly overlapping papers have recently investigated the $125 \mathrm{GeV}$ Higgs-like excess in the context of composite Higgs [16], supersymmetric Higgs [17-28], and multi-Higgs models [29-32]; see also [33-41]. For earlier related work, see [42-46].

Of course, at this stage the limited statistical power of the current Higgs data does not allow us to make a strong statement about any new physics scenario. Nevertheless, in several cases we are able to identify non-trivial regions of the parameter space that are disfavored at $95 \% \mathrm{CL}$. Repeating this analysis with future data may allow us, in the best case scenario, to pinpoint departures of the Higgs couplings from the SM predictions. That would not only provide evidence of new physics, but also some information about its scale, thereby supplying important hints about the nature of the fundamental theory at the electroweak scale.

The paper is organized as follows. In the next section we define the effective action for a Higgs boson interacting with the SM fields and identify the relevant parameters that are being constrained by the present data. In section 3 we discuss the data and provide the combined best-fit of the ATLAS, CMS, and Tevatron Higgs results. We then show the resulting constraints on the parameters of the Higgs effective action. In section 4 we then study simplified models with scalar top partners, relevant for the MSSM as well. Section 5 focuses on fermionic top partners which show up in many composite Higgs and Little Higgs models. Several representative examples are discussed. Section 6 discusses some implications of theories with 2 Higgs particles. We conclude in section 7. 


\section{Formalism}

We begin by defining a convenient framework to describe LHC and Tevatron Higgs phenomenology. We define an effective theory at the scale $\mu \sim m_{h}$, which describes the couplings of a single Higgs boson, $h$, to the SM gauge bosons and fermions. Keeping dimension 5 operators and writing only couplings to the heaviest fermions we have,

$$
\begin{aligned}
\mathcal{L}_{\text {eff }}= & c_{V} \frac{2 m_{W}^{2}}{v} h W_{\mu}^{+} W_{\mu}^{-}+c_{V} \frac{m_{Z}^{2}}{v} h Z_{\mu} Z_{\mu}-c_{b} \frac{m_{b}}{v} h \bar{b} b-c_{\tau} \frac{m_{\tau}}{v} h \bar{\tau} \tau \\
& +c_{g} \frac{\alpha_{s}}{12 \pi v} h G_{\mu \nu}^{a} G_{\mu \nu}^{a}+c_{\gamma} \frac{\alpha}{\pi v} h A_{\mu \nu} A_{\mu \nu} .
\end{aligned}
$$

Here $v=246 \mathrm{GeV}$, and $G_{\mu \nu}^{a}$ and $A_{\mu \nu}$ are the field strengths of the gluon and photon, respectively. The fact that the same parameter $c_{V}$ controls the coupling to $\mathrm{W}$ and $\mathrm{Z}$ boson follows from the assumption that these couplings respect, to a good approximation, custodial symmetry, as strongly suggested by electroweak precision observables. We note that the Higgs could decay to particles from beyond the SM, e.g. to invisible collider-stable particles, but we will not discuss this possibility here. We further note that while a single Higgs is kept at low energy, the above may describe multi-Higgs models, as long as there is a sizable splitting between the lightest and the remaining Higgs fields. We study such a possibility in more detail in section 6 .

In (2.1), the top quark has been integrated out, contributing at 1-loop to $c_{g}$ and $c_{\gamma}$ as

$$
c_{g}\left(\tau_{t}\right)=c_{t} A_{f}\left(\tau_{t}\right), \quad c_{\gamma}\left(\tau_{t}\right)=\frac{2 c_{t}}{9} A_{f}\left(\tau_{t}\right), \quad A_{f}(\tau)=\frac{3}{2 \tau^{2}}[(\tau-1) f(\tau)+\tau],
$$

where $\tau_{t}=m_{h}^{2} / 4 m_{t}^{2}, c_{t}$ is the ratio of the top-Higgs Yukawa coupling to the SM one, and

$$
f(\tau)=\left\{\begin{array}{ll}
\arcsin ^{2} \sqrt{\tau} & \tau \leq 1 \\
-\frac{1}{4}\left[\log \frac{1+\sqrt{1-\tau^{-1}}}{1-\sqrt{1-\tau^{-1}}}-i \pi\right]^{2} & \tau>1
\end{array} .\right.
$$

For $m_{h}^{2} \ll 4 m_{t}^{2}$ one finds, $f(\tau) \simeq \tau(1+\tau / 3)$, which is a very good approximation for $m_{h} \lesssim 130 \mathrm{GeV}$. Consequently, for the SM with a light Higgs boson matched to our effective theory at 1-loop we have

$$
c_{V, \mathrm{SM}}=c_{b, \mathrm{SM}}=1, \quad c_{g, \mathrm{SM}} \simeq 1, \quad c_{\gamma, \mathrm{SM}} \simeq 2 / 9 .
$$

The decay widths of the Higgs relative to the SM predictions are modified approximately as,

$$
\begin{aligned}
\frac{\Gamma(h \rightarrow b \bar{b})}{\Gamma_{S M}(h \rightarrow b \bar{b})}=\left|c_{b}\right|^{2}, & \frac{\Gamma\left(h \rightarrow W W^{*}\right)}{\Gamma_{S M}\left(h \rightarrow W W^{*}\right)}=\frac{\Gamma\left(h \rightarrow Z Z^{*}\right)}{\Gamma_{S M}\left(h \rightarrow Z Z^{*}\right)}=\left|c_{V}\right|^{2}, \\
\frac{\Gamma(h \rightarrow g g)}{\Gamma_{S M}(h \rightarrow g g)} \simeq\left|c_{g}\right|^{2}, & \frac{\Gamma(h \rightarrow \gamma \gamma)}{\Gamma_{S M}(h \rightarrow \gamma \gamma)}=\left|\frac{\hat{c}_{\gamma}}{\hat{c}_{\gamma, S M}}\right|^{2},
\end{aligned}
$$

where $\hat{c}_{\gamma}$ includes also the one-loop contribution due to the triangle diagram with the $\mathrm{W}$ boson, ${ }^{1}$

$$
\hat{c}_{\gamma}\left(\tau_{t}, \tau_{W}\right)=c_{\gamma}\left(\tau_{t}\right)-\frac{c_{V}}{8 \tau_{W}^{2}}\left[3\left(2 \tau_{W}-1\right) f\left(\tau_{W}\right)+3 \tau_{W}+2 \tau_{W}^{2}\right],
$$

\footnotetext{
${ }^{1}$ There are additional one-loop contributions to $c_{\gamma}$ and $c_{g}$ from light quarks that are left out in this discussion, but are included in the analyses below.
} 
where $\tau_{W}=m_{h}^{2} / 4 m_{W}^{2}$. For $m_{h}=125 \mathrm{GeV}$ one finds $\hat{c}_{\gamma} \simeq c_{\gamma}-1.04 c_{V}$, and thus $\hat{c}_{\gamma, S M} \simeq$ -0.81 .

More generally, the 1-loop contribution to $c_{g}$ from an additional fermion in the fundamental representation of $\mathrm{SU}(3)_{C}$ and coupled to the Higgs via the Yukawa coupling $y_{f} h \bar{f} / \sqrt{2}$ is simply given by eq. (2.2) with $c_{t} \rightarrow\left(v y_{f} / \sqrt{2} m_{f}\right)$ and $\tau_{t} \rightarrow \tau_{f}$, while for an $\mathrm{SU}(3)_{C}$ fundamental scalar,

$$
\delta c_{g}\left(\tau_{s}\right)=\frac{1}{4} \sum_{s} g_{h s s} A_{s}\left(\tau_{s}\right), \quad g_{h s s}=\frac{1}{2} \frac{v}{m_{s}^{2}} \frac{\partial m_{s}^{2}}{\partial v}, \quad A_{s}(\tau)=\frac{3}{\tau^{2}}[f(\tau)-\tau] .
$$

For the photon coupling we have

$$
\delta c_{\gamma}\left(\tau_{f, s}\right)=\frac{Q_{f, s}^{2}}{2} \delta c_{g}\left(\tau_{f, s}\right),
$$

where $Q_{f, s}$ is the electric charge of the scalar or fermion. More general expressions can be found e.g in $[47,48]$. Note that in the limit $\tau \rightarrow 0, A_{f, s}(\tau) \rightarrow 1$, and the scalar contribution becomes $1 / 4$ that of the fermion. In fact, the $\tau \rightarrow 0$ limit is equivalent to approximating $c_{g}$ and $c_{\gamma}$ using the 1-loop beta function [49, 50], which explains the relative factor $1 / 4$.

As discussed in the introduction, the most significant constraints on the effective theory are obtained by studying several independent Higgs decay channels. The five most constraining channels to date are $h \rightarrow Z Z^{*}, h \rightarrow W W^{*}, h \rightarrow \gamma \gamma, p p \rightarrow h V \rightarrow b \bar{b} V$ and $p p \rightarrow h j j \rightarrow \gamma \gamma j j$. The Higgs production mechanism in the first three channels is dominated by the gluon fusion process which scales as $c_{g}^{2}$. The $b \bar{b}$ channel is dominated by associate production which scales as $c_{V}^{2}$. Thus, the relevant Higgs event rates scale as,

$$
\begin{aligned}
R_{V V} & \equiv \frac{\sigma(p p \rightarrow h) \operatorname{Br}\left(h \rightarrow V V^{*}\right)}{\sigma_{S M}(p p \rightarrow h) \operatorname{Br}_{S M}\left(h \rightarrow V V^{*}\right)} \simeq\left|\frac{c_{g} c_{V}}{C_{\mathrm{tot}}}\right|^{2}, \\
R_{\gamma \gamma} & \equiv \frac{\sigma(p p \rightarrow h) \operatorname{Br}(h \rightarrow \gamma \gamma)}{\sigma_{S M}(p p \rightarrow h) \operatorname{Br}_{S M}(h \rightarrow \gamma \gamma)} \simeq\left|\frac{c_{g} \hat{c}_{\gamma}}{\hat{c}_{\gamma, S M} C_{\mathrm{tot}}}\right|^{2}, \\
R_{b \bar{b} V} & \equiv \frac{\sigma(p \bar{p} \rightarrow h V) \operatorname{Br}(h \rightarrow b \bar{b})}{\sigma_{S M}(p \bar{p} \rightarrow h V) \operatorname{Br}_{S M}(h \rightarrow b \bar{b})} \simeq\left|\frac{c_{V} c_{b}}{C_{\mathrm{tot}}}\right|^{2},
\end{aligned}
$$

where $\left|C_{\text {tot }}\right|^{2}=\Gamma_{\text {tot }} / \Gamma_{\text {tot }}^{S M}$. The approximation holds assuming the Higgs production remains dominated by the gluon fusion subprocess. More precise relations are used in our fits.

The $\gamma \gamma j j$ channel is slightly more complex, since it receives comparable contributions from the gluon fusion and VBF production channels:

$$
\begin{aligned}
R_{\gamma \gamma j j} & \equiv \frac{\sigma(p p \rightarrow h j j) \operatorname{Br}(h \rightarrow \gamma \gamma)}{\sigma_{S M}(p p \rightarrow h j j) \operatorname{Br}_{S M}(h \rightarrow \gamma \gamma)} \\
& =\frac{\sigma_{g g f}^{S M} \cdot \epsilon_{g g f} \cdot\left|c_{g}\right|^{2}+\sigma_{V B F}^{S M} \cdot \epsilon_{V B F} \cdot\left|c_{V}\right|^{2}}{\sigma_{g g f}^{S M} \cdot \epsilon_{g g f}+\sigma_{V B F}^{S M} \cdot \epsilon_{V B F}} \cdot\left|\frac{\hat{c}_{\gamma}}{\hat{c}_{\gamma, S M} C_{\mathrm{tot}}}\right|^{2}
\end{aligned}
$$

where $\epsilon_{g g f}$ and $\epsilon_{V B F}$ are the efficiencies to pass the selection cuts in the gluon fusion and VBF production modes, respectively. In the SM, the gluon fusion mode contributes about 
$1 / 3$ that of the VBF production mode to the $2 \gamma 2 j$ final state studied by CMS [8,9], but it may become more important in models where the gluon fusion cross section is enhanced relative to the $\mathrm{VBF}$ one. ${ }^{2}$

\section{Constraints from the LHC and Tevatron}

Recently, LHC and Tevatron have reported the results of Higgs searches in several channels. Here we focus on the following channels: $h \rightarrow \gamma \gamma$ and $p p \rightarrow h j j \rightarrow \gamma \gamma j j[5,8,9], h \rightarrow$ $Z Z^{*} \rightarrow 4 l[6,10], h \rightarrow W W^{*} \rightarrow 4 l[15], V h \rightarrow V b \bar{b}$ [14] channels, which are currently the most sensitive ones for $115<m_{h}<130 \mathrm{GeV}$.

Both LHC and Tevatron observe an excess of events that (inconclusively) indicate the existence of a Higgs boson with mass in the $124-126 \mathrm{GeV}$ range. The largest excess comes from CMS in the rate $R_{\gamma \gamma j j}$, and hints towards a cross-section which is larger then in the SM. In the diphoton channel, both CMS and ATLAS observe a rate $R_{\gamma \gamma}$ which is consistent with the SM. The Tevatron sees a small excess in $R_{b \bar{b}}$, as compared to the SM higgs. Consequently, as will be shown below, combining the results together points to a production cross-section and branching fractions consistent with that predicted by the SM. It remains to be seen whether with better statistics and improved understanding of the systematics, the results will remain consistent with the SM prediction, or otherwise converge on a rate deviating from that predicted by the SM.

In order to constrain the couplings of the effective theory $-c_{g}, c_{\gamma}, c_{V}, c_{b}$ and $c_{\tau}$ in eq. (2.1) - it is crucial to analyze several Higgs production and decay modes. Following the discussion above, we focus on the channels which are the most constraining, i.e eqs. (2.9)(2.12). Since one of the production modes (gluon fusion) and one of the decay modes $(\gamma \gamma)$ are loop-induced, these constraints are very sensitive to heavy particles beyond the SM that may play a role in solving the fine tuning problem, leading to interesting conclusions on new physics and naturalness.

In figure 1 we show the results of the combined best fit value of $\hat{R} \equiv \sigma / \sigma_{\mathrm{SM}}$, assuming gaussian statistics, for each of the analyzed channels separately and for the combination of all channels, for Higgs mass between $120 \mathrm{GeV}$ and $130 \mathrm{GeV}$. The bands indicate the $1 \sigma$ uncertainty. Since the CMS experiment does not provide the values of $R_{\gamma \gamma j j}$ for $m_{H}=$ $120-130 \mathrm{GeV}$, we calculated the best fit for the rates in the channel, which we show in figure 1. More specifically, we repeat the analyses, computing the likelihood functions. We use background and signal modeling given by the experiments, normalizing the signal to the reported values. For the results shown here, we do not take into account the systematic effects which are expected to be significant in the dijet channel of the diphoton analysis.

In figure 2 we use our results of figure 1 to place constraints on the effective theory assuming $m_{h}=125 \mathrm{GeV}$. We show two dimensional constraints on $\delta c_{g}=c_{g}-c_{g, \mathrm{SM}}$, $\delta c_{\gamma}=c_{\gamma}-c_{\gamma, \mathrm{SM}}$ and $c_{V}$ for various model assumptions. Shown are the $1 \sigma$ allowed regions for $R_{\gamma \gamma}$ (purple), $R_{Z Z}$ (blue), $R_{W W}$ (light grey), $R_{\gamma \gamma j j}$ (beige), $R_{b \bar{b}}$ (orange). The green region gives the allowed region at $95 \%$ CL for the combination of all channels. In figure $2 \mathrm{a}$ we allow only the Higgs couplings to gluons and photons to change while keeping the other

\footnotetext{
${ }^{2}$ We thank Yevgeny Kats for pointing this out to us.
} 

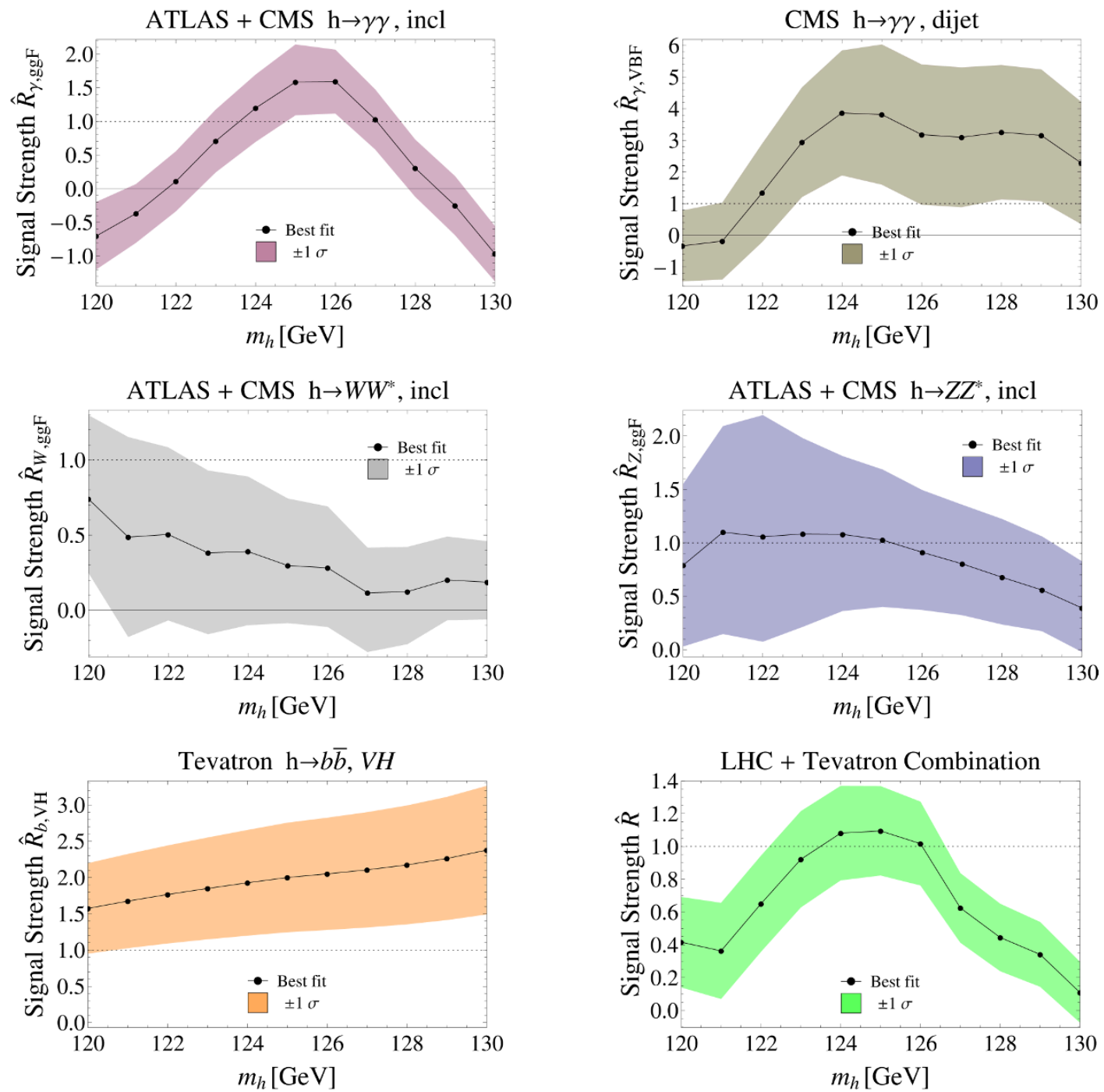

Figure 1. Best-fit values and $1 \sigma$ bands for the rates $R_{\gamma \gamma}, R_{\gamma \gamma j j}, R_{W W}, R_{Z Z}$, and $R_{b \bar{b}}$ for a Higgs mass between $120 \mathrm{GeV}$ and $130 \mathrm{GeV}$. We also show the combination of all the channels (bottomright). In all but the CMS dijet measurement, the results are computed using the reported results and assuming gaussian statistics. For the CMS dijet, the best fits are derived by repeating the analysis reported in $[8,9]$, not taking systematic uncertainties into account. The results in the dijet mode are found to be conservative.

couplings at the SM values. In the remaining plots of figure 2 we keep $\delta c_{\gamma} / \delta c_{g}=2 / 9$ fixed, while varying the other couplings. That ratio is conserved when top partners with the same charge and color as the top are introduced.

In the next three sections, we study various models that allow for an improvement in the fine-tuning of the Higgs mass. Our goal is to keep the discussion quite general, and we therefore consider simplified models that capture different paradigms showing up in many 

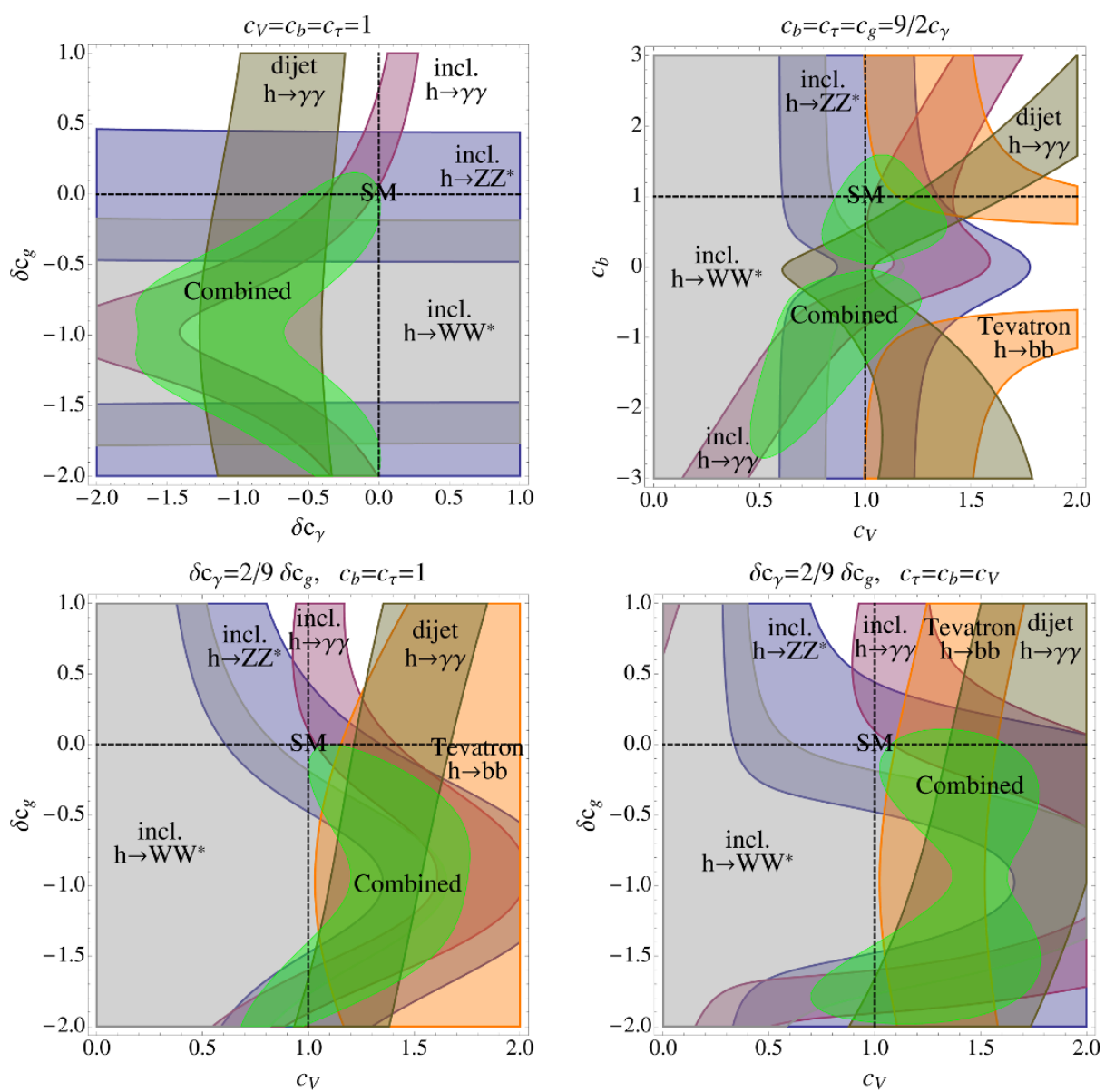

Figure 2. The allowed parameter space of the effective theory given in eq. (2.1), derived from the LHC and Tevatron constraints for $m_{h}=125 \mathrm{GeV}$. We display the $1 \sigma$ allowed regions for the rates in eqs. (2.9)-(2.12): $R_{\gamma \gamma}$ (purple), $R_{Z Z}$ (blue), $R_{W W}$ (light grey), $R_{\gamma \gamma j j}$ (beige), and $R_{b \bar{b}}$ (orange). The "Combined" region (green) shows the $95 \%$ CL allowed region arising from all channels. The crossing of the dashed lines is the SM point. The top left plot characterizes models in which loops containing beyond the SM fields contribute to the effective 5-dimensional $h G_{\mu \nu}^{a} G_{\mu \nu}^{a}$ and $h A_{\mu \nu} A_{\mu \nu}$ operators, while leaving the lower-dimension Higgs couplings in eq. (2.1) unchanged relative to the SM prediction. The top right plot characterizes composite Higgs models and can be compared to [76] and [77]. The lower plots characterize top partner models where only scalars and fermions with the same charge and color as the top quark contribute to the effective 5-dimensional operators, which implies the relation $\delta c_{\gamma}=(2 / 9) \delta c_{g}$. The results are shown for 2 different sets of assumptions about the lower-dimension Higgs couplings that can be realized in concrete models addressing the Higgs naturalness problem.

models that solve the fine-tuning problem. Each of the models is then mapped on to the effective theory, eq. (2.1), and the constraints derived above are used to place bounds on the specific scenarios. Throughout the paper, we use the results of figure 1, except for the plots which assume $m_{h}=125 \mathrm{GeV}$, for which we use the (somewhat stronger) constraints on the dijet rate, $R_{\gamma \gamma j j}$, reported by CMS in the MVA analysis [8, 9] . 


\section{Models with scalar top partners}

\subsection{One scalar}

We start our exploration with the simple toy model of a single scalar top partner. Consider a scalar $\tilde{t}$ with electric charge $2 / 3$ and transforming in the fundamental representation under the $\mathrm{SU}(3)$ color. At the renormalizable level, the top sector mass and interaction terms can be parametrized as

$$
\mathcal{L}_{\text {stop }}=-\left(y H Q t^{c}+\text { h.c. }\right)-|\tilde{t}|^{2}\left(M^{2}+\lambda|H|^{2}\right) .
$$

Here $Q=(t, b)$ is the 3rd generation quark doublet, $t^{c}$ is the $\mathrm{SU}(2)_{W}$ singlet top and $H$ is the Higgs doublet. In the unitary gauge $H=(0,(v+h) / \sqrt{2})$ and $|H|=(v+h) / \sqrt{2}$, where $v=246 \mathrm{GeV}$ and $h$ is the canonically normalized Higgs boson field. It follows that $m_{\tilde{t}}^{2}=M^{2}+\lambda v^{2} / 2$. The quadratic divergent top contribution to the Higgs mass is canceled by the scalar partner when the coupling $\lambda$ is related to the top Yukawa coupling by

$$
\lambda=2 y^{2} .
$$

Note this is different than in minimal supersymmetry where 2 scalar partners with $\lambda \simeq y^{2}$ play a role in canceling the top quadratic divergence.

For $m_{\tilde{t}} \gg m_{h} / 2$, using eqs. (2.7), (2.8) one finds the scalar partner contribution to the effective dimension 5 operator,

$$
\frac{c_{g}}{c_{g, \mathrm{SM}}}=\frac{c_{\gamma}}{c_{\gamma, \mathrm{SM}}} \simeq 1+\lambda \frac{v^{2}}{8 m_{\tilde{t}}^{2}}=1+\frac{m_{t}^{2}}{2 m_{\tilde{t}}^{2}}, \quad \quad c_{V}=c_{b}=1
$$

The last equality holds when eq. (4.2) is satisfied. Thus, if the scalar top partner is soley responsible for the cancellation of the top quadratic divergence, then the gluon fusion rate is always enhanced, while the diphoton rate is slightly suppressed for realistic $m_{\tilde{t}}$ (due to interference with the negative $\mathrm{W}$ loop contribution). This is unlike the MSSM where both enhancement and suppression of the gluon fusion can be realized within the realistic parameter space (see below).

In figure 3 we show the 95\% CL allowed region for $m_{\tilde{t}}$ as a function of the Higgs mass (left), along with the $1 \sigma$ bounds for $m_{h}=125 \mathrm{GeV}$ (right). We see that model independently, a single scalar top partner lighter than $240 \mathrm{GeV}$ is excluded, if indeed the LHC and Tevatron signals correspond to a $125 \mathrm{GeV}$ Higgs boson, as hinted by the data.

\subsection{Two scalars (MSSM)}

Consider the system of 2 scalar top partners $\tilde{t}, \tilde{t}^{c}$, one for the left-handed top and one for the right-handed top, with the mass terms of the form

$$
-\mathcal{L}_{\text {stop }}=|\tilde{t}|^{2}\left(\tilde{m}^{2}+y^{2}|H|^{2}\right)+\left|\tilde{t}^{c}\right|^{2}\left(\tilde{m}_{c}^{2}+y^{2}|H|^{2}\right)+y|H| X_{t}\left(\tilde{t}^{c}+\text { h.c. }\right),
$$

where $y$ is the top Yukawa coupling, as in eq. (4.1). This is equivalent to the stop sector of the MSSM in the decoupling limit $\left(M_{A} \gg M_{Z}\right)$ and neglecting the (sub-leading) D-terms 

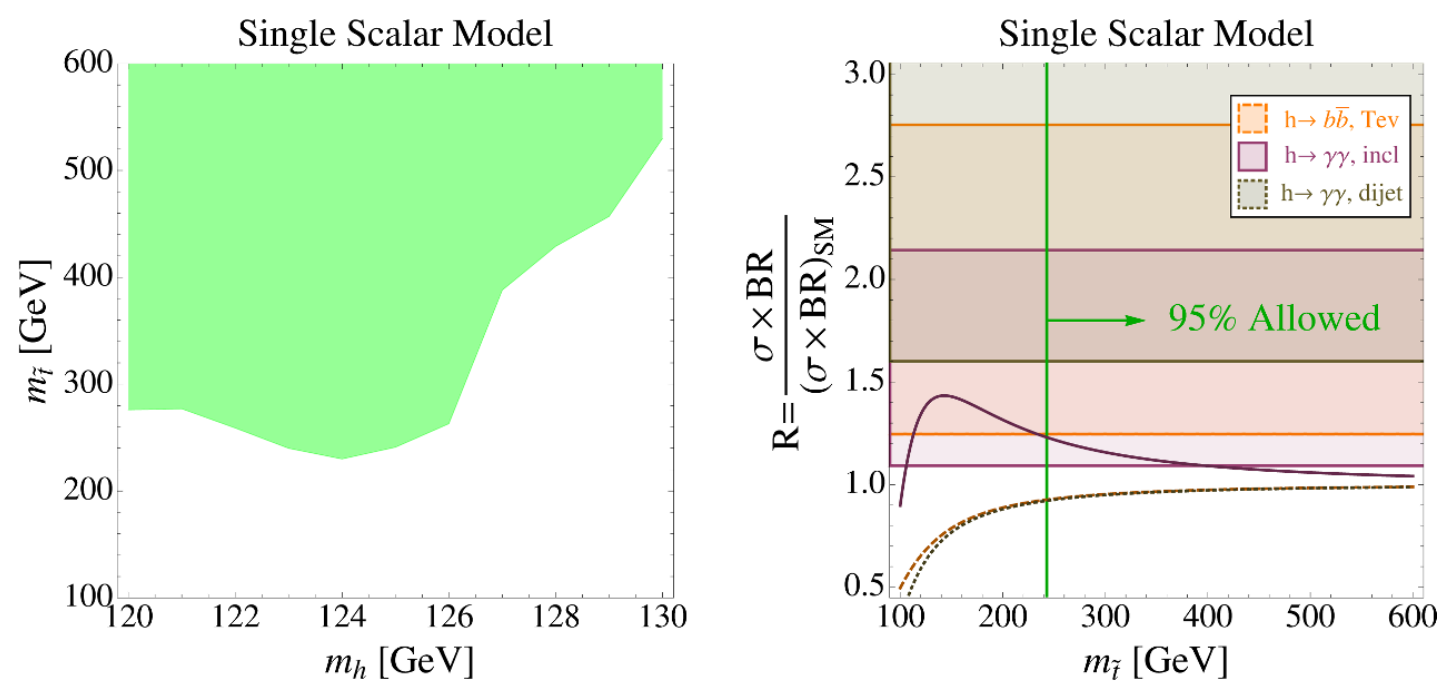

Figure 3. Left: Favored region, $95 \% \mathrm{CL}$, in the $m_{\tilde{t}}-m_{h}$ plane, derived from the combination of all search channels, for the one-scalar model described in section 4.1. Right: Constraints for $m_{h}=125 \mathrm{GeV}$. The three bands show the $1 \sigma$ allowed regions: $R_{\gamma \gamma}$ (purple), $R_{b \bar{b}}$ (orange), $R_{\gamma \gamma j j}$ (beige). The three curves show the theoretical predictions as a function of $m_{\tilde{t}}: R_{\gamma \gamma}$ (solid-purple), $R_{b \bar{b}}$ (dashed-orange), and $R_{\gamma \gamma j j}$ (dotted-beige). Only 3 channels are shown, but all channels are included. The region to the right of the green line at $m_{\tilde{t}}=240 \mathrm{GeV}$ shows the $95 \% \mathrm{CL}$ experimental allowed region.

contribution to the stop masses. Here the contributions of both scalars sum to cancel the quadratic divergence from the top quark. The left-handed and right-handed stops mix in the presence of $X_{t}$, which in the MSSM is given by $X_{t}=\left|A_{t}-\mu \cot \beta\right|$. See e.g [53].

Denoting the two mass eigenvalues by $m_{\tilde{t}_{i}}$, and the left-right mixing angle by $\theta_{t}$, one has

$$
m_{t} X_{t}=\frac{1}{2}\left(m_{\tilde{t}_{2}}^{2}-m_{\tilde{t}_{1}}^{2}\right) \sin 2 \theta_{t}
$$

where, by convention, $m_{\tilde{t}_{1}} \leq m_{\tilde{t}_{2}}$. For $m_{\tilde{t}_{i}} \gg m_{h} / 2$, integrating out the stops shifts the effective dimension- 5 operators as

$$
\frac{c_{g}}{c_{g, \mathrm{SM}}}=\frac{c_{\gamma}}{c_{\gamma, \mathrm{SM}}}=1+\frac{1}{4}\left(\frac{m_{t}^{2}}{m_{\tilde{t}_{1}}^{2}}+\frac{m_{t}^{2}}{m_{\tilde{t}_{2}}^{2}}-\frac{m_{t}^{2} X_{t}^{2}}{m_{\tilde{t}_{1}}^{2} m_{\tilde{t}_{2}}^{2}}\right) .
$$

For zero mixing, the stops always interfere constructively with the top contribution (destructively with the $W$-contribution to $\hat{c}_{\gamma}$ ), but once $X_{t}$ becomes comparable to stop masses an enhancement of $c_{g}$ becomes possible. A significant shift of the gluon fusion and diphoton widths is possible if at least one of the stop mass eigenvalues is close to the top mass, or if the mixing is very large.

In figure 4 we illustrate the impact of the LHC and Tevatron Higgs data on the parameter space of the 2-scalar-partner model. The left plot shows the allowed region in the $m_{\tilde{t}_{1}}-X_{t} / m_{\tilde{t}_{2}}$ plane, assuming that $m_{\tilde{t}_{2}}^{2}$ is large enough so that the heavier stop eigenstate does not contribute to the effective operators (that is, dropping the second term in the 

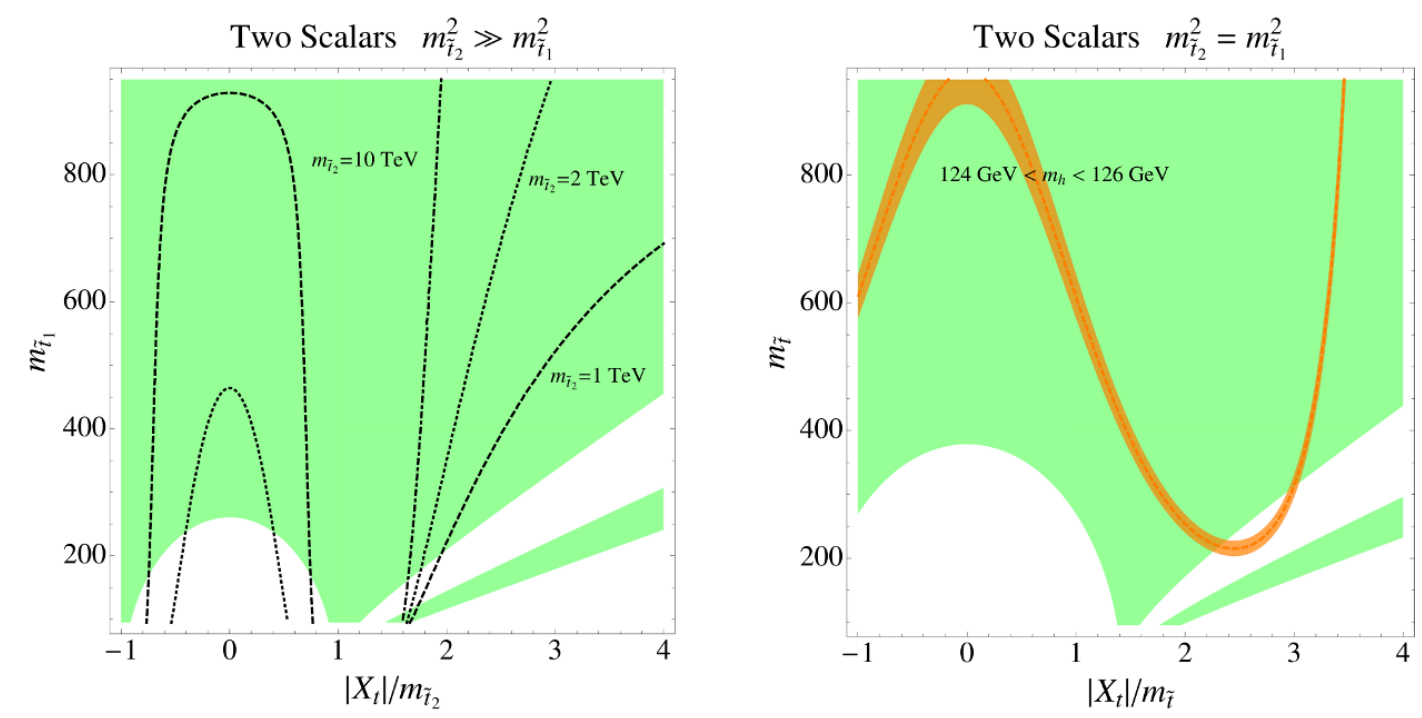

Figure 4. Left: The favored region at $95 \% \mathrm{CL}$ for $m_{h}=125 \mathrm{GeV}$, derived from the combination of all search channels, in the two scalar model with $m_{\tilde{t}_{2}} \gg m_{\tilde{t}_{1}}$. Also shown are contours of constant $m_{h}=125 \mathrm{GeV}$ assuming the 1-loop MSSM relation between Higgs and stop masses, for $m_{\tilde{t}_{2}}=1,2$, and $10 \mathrm{TeV}$. Right: Same for $m_{\tilde{t}_{2}}=m_{\tilde{t}_{1}}$. Also shown is a band corresponding to $124 \mathrm{GeV}$ $<m_{h}<16 \mathrm{GeV}$ assuming the 1-loop MSSM relation between Higgs and stop masses. Additional, model-dependent, bounds on stops from direct searches are not shown.

bracket in eq. (4.6)). For no mixing, $X_{t}^{2} / m_{\tilde{t}_{2}}^{2}=0$, the lower bound on the lightest stop is $\sim 250 \mathrm{GeV}$.

In both scenarios, for just right amount of mixing, that is $X_{t} / m_{\tilde{t}_{2}} \simeq 1$ for $m_{\tilde{t}_{2}}^{2} \gg m_{\tilde{t}_{1}}^{2}$ and $\left|X_{t}\right| / m_{\tilde{t}_{2}} \simeq \sqrt{2}$ for $m_{\tilde{t}_{2}}^{2}=m_{\tilde{t}_{1}}^{2}$, the scalar partners contribution to $c_{g}$ and $c_{\gamma}$ can vanish, even for very light stops. This may be relevant for models that require a light stop, such as electroweak baryogenesis [54-56]. For illustration, on the left plot of figure 4 we show contours of constant $m_{h}=125 \mathrm{GeV}$, for $m_{\tilde{t}_{2}}=1,2$, and $10 \mathrm{TeV}$, while on the right plot we show the region where $124 \mathrm{GeV}<m_{h}<126 \mathrm{GeV}$. We note that that used the one-loop formula for the Higgs mass in the MSSM, therefore these contours should be considered illustrative only. As a final remark, we comment that additional bounds on stops exist from direct searches. These bounds are however model dependent, in particular strongly depending on the stop decay branching fractions, and therefore we do not display them.

\section{Models with a fermionic top partner}

We move to the case of one fermionic top partner. Consider the SM model extended by a vector-like quark pair $\left(T, T^{c}\right)$ in the $\mathbf{1}_{2 / 3}$ representation under $\mathrm{SU}(2)_{W} \times \mathrm{U}(1)_{Y}$. Fermionic partners cannot cancel the top quadratic divergence if the effective Lagrangian describing their interactions with the Higgs is renormalizable. Therefore in this case we need to consider a more general effective Lagrangian for the top sector that includes non- 
renormalizable interactions,

$$
-\mathcal{L}_{\text {top }}=y_{1}\left(|H|^{2}\right) H Q t^{c}+y_{2}\left(|H|^{2}\right) H Q T^{c}+M_{1}\left(|H|^{2}\right) T t^{c}+M_{2}\left(|H|^{2}\right) T T^{c}+\text { h.c. } .
$$

We allow the vacuum expectation value of the Higgs doublet, $\hat{v}$, to be different from the electroweak scale $v=246 \mathrm{GeV}$, which may happen if the Higgs effective interactions with $\mathrm{W} / \mathrm{Z}$ bosons are also non-renormalizable and corresponds to $c_{V} \neq 1$ (this is in fact the case in Little Higgs and composite Higgs models). We assume that all mass and Yukawa couplings are functions of $|H|^{2}$ and can be expanded in powers of $|H|^{2} / M^{2}$ where $M$ is the mass scale of the heavy top quark. Up to order $|H|^{2} / M^{2}$ they can be parametrized as

$$
\begin{aligned}
& y_{1}\left(|H|^{2}\right)=y_{1}\left(1-d_{1} \frac{|H|^{2}}{M^{2}}\right)+\mathcal{O}\left(\frac{|H|^{4}}{M^{4}}\right), \\
& y_{2}\left(|H|^{2}\right)=y_{2}\left(1-d_{2} \frac{|H|^{2}}{M^{2}}\right)+\mathcal{O}\left(\frac{|H|^{4}}{M^{4}}\right), \\
& M_{1}\left(|H|^{2}\right)=c_{1} M \frac{|H|^{2}}{M^{2}}+\mathcal{O}\left(\frac{|H|^{4}}{M^{4}}\right), \\
& M_{2}\left(|H|^{2}\right)=M\left(1-c_{2} \frac{|H|^{2}}{M^{2}}\right)+\mathcal{O}\left(\frac{|H|^{4}}{M^{4}}\right) .
\end{aligned}
$$

Above, we used the freedom to rotate $t^{c}$ and $T^{c}$ such that $M_{1}$ starts at $\mathcal{O}\left(|H|^{2}\right)$. In terms of these parameters $m_{\text {top }} \simeq y_{1} \hat{v} / \sqrt{2}$ while $m_{T} \simeq M$. For the cancellation of the quadratic divergences in the Higgs mass term, one straightforwardly finds,

$$
c_{2}=\frac{y_{1}^{2}+y_{2}^{2}}{2} .
$$

This relation may arise naturally in models where the Higgs is realized as a pseudoGoldstone boson of a spontaneously broken approximate global symmetry.

Following the discussion above eq. (2.7) and integrating out the top sector, one finds for the effective Higgs coupling to gluons and photons shifts as

$$
\frac{c_{g}}{c_{g, \mathrm{SM}}}=\frac{c_{\gamma}}{c_{\gamma, \mathrm{SM}}} \simeq \frac{v}{\hat{v}}\left[1-\frac{\hat{v}^{2}}{M^{2}}\left(d_{1}+c_{2}+\frac{c_{1} y_{2}}{y_{1}}\right)\right] .
$$

We see that several parameters of the effective Lagrangian enter the modification of effective Higgs coupling to gluons and photons. Above, $y_{1}$ can be eliminated in favor of the top mass, and $c_{2}$ can be eliminated using the condition eq. (5.6). This still leaves 4 free parameters: $d_{1}, y_{2}, \hat{v} / v$ and $M$. Thus, in full generality, we cannot predict the magnitude, or even the sign of the correction to the Higgs rate merely by demanding cancellation of quadratic divergences. ${ }^{3}$ However, concrete realizations of Little Higgs and composite Higgs models often imply additional relations between the effective theory parameters, in which case the set-up becomes more predictive. Below we study several predictive patterns of effective theory parameters that arise in popular Little Higgs and composite Higgs models.

\footnotetext{
${ }^{3}$ In composite Higgs models under certain conditions one can argue that the gluon fusion and diphoton decay rate cannot be enhanced [59].
} 

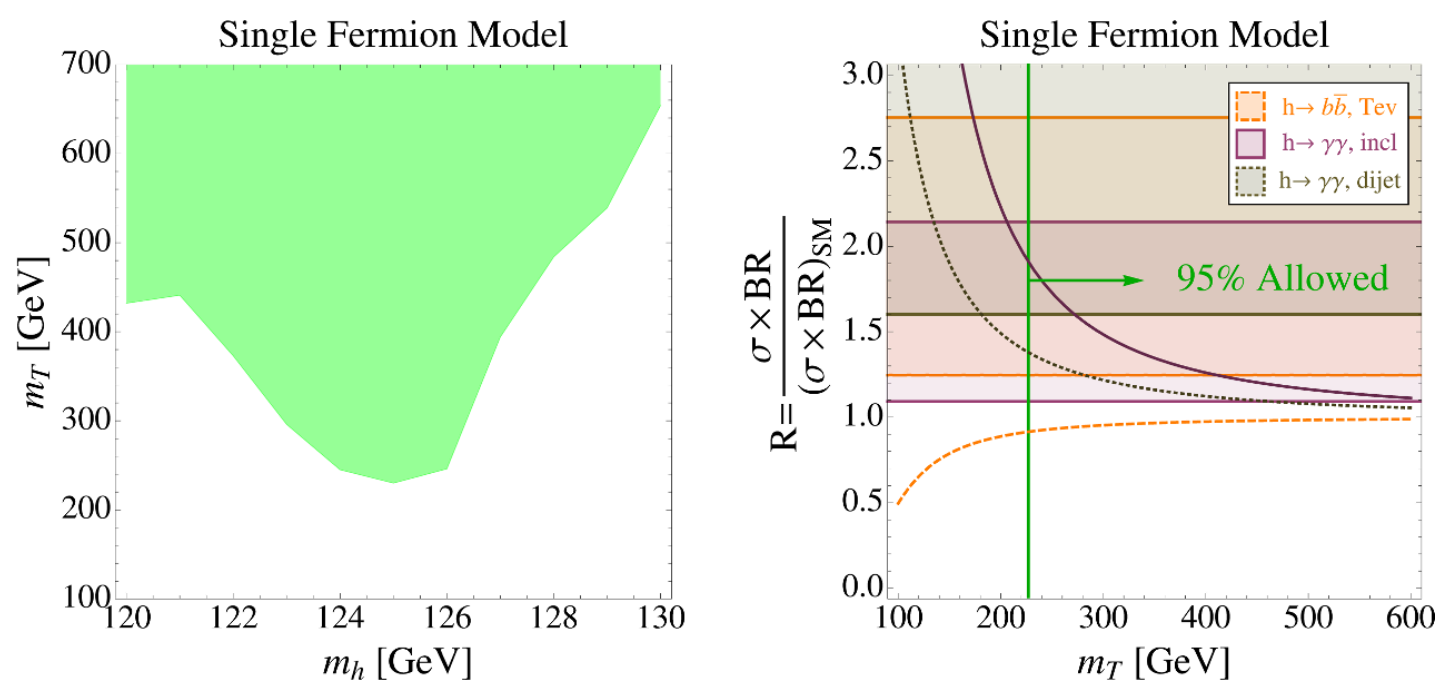

Figure 5. Left: Favored region, 95\% CL, in the $m_{T}-m_{h}$ plane, derived from the combination of all search channels, for the single-fermion, no-mixing model described in section 5.1. Right: Constraints assuming $m_{h}=125 \mathrm{GeV}$. The three bands show the $1 \sigma$ allowed regions: $R_{\gamma \gamma}$ (purple), $R_{b \bar{b}}$ (orange), $R_{\gamma \gamma j j}$ (beige). The three curves show the theoretical predictions as a function of $m_{T}$ : $R_{\gamma \gamma}$ (solid-purple), $R_{b \bar{b}}$ (dashed-orange) and $R_{\gamma \gamma j j}$ (dotted-beige). Only 3 channels are shown, but all channels are included. The region to the right of the green line at $m_{T}=220 \mathrm{GeV}$ shows the 95\% CL experimental (combined) allowed region.

\section{$5.1 \quad$ No mixing}

First, we will restrict the parameter space by demanding that the SM top does not mix with its partners, $c_{1}=y_{2}=0$, and $c_{2}=y_{1}^{2} / 2 \simeq m_{t}^{2} / v^{2}$. This situation occurs in Little Higgs with T-parity $[57,58]$. Furthermore, we assume that the Higgs coupling to the SM fields is not modified at $\mathcal{O}\left(v^{3}\right)$, thus $\hat{v}=v$ and $d_{1}=0$. Under these assumptions one finds

$$
\frac{c_{g}}{c_{g, \mathrm{SM}}}=\frac{c_{\gamma}}{c_{\gamma, \mathrm{SM}}} \simeq 1-\frac{m_{t}^{2}}{m_{T}^{2}}, \quad c_{V}=c_{b}=1 .
$$

Hence in this scenario, much as in the case of one scalar partner, the departure of the Higgs rates from the SM can be described by one parameter: the ratio of the top mass to its partner mass. The gluon width, and in consequence the dominant Higgs production mode, is reduced. On the other hand, the Higgs partial width into $W W$ and $Z Z$ are unchanged, while the partial width in the $\gamma \gamma$ channel is significantly changed only when $m_{T} \sim m_{t}$. In figure 5 we present the constraints on $m_{T}$ from the LHC and Tevatron Higgs data. In the left plot we show the $95 \% \mathrm{CL}$ allowed region, in the $m_{h}-m_{T}$ plane. The right plot shows the constraints assuming a $125 \mathrm{GeV}$ Higgs. As can be seen, $m_{T} \lesssim 220 \mathrm{GeV}$ is disfavored in this case.

\subsection{Universal suppression}

Consider now a frequently occurring situation when all the Higgs rates are suppressed by a universal factor depending on the compositeness scale $f$. To be specific, consider the top 
sector interacting with a pseudo-Goldstone Higgs as

$$
-\mathcal{L}_{\text {top }}=y f \sin (|H| / f) t t^{c}+y f \cos (|H| / f) T t^{c}+M^{\prime} T T^{c}+\text { h.c. } .
$$

The top partner mass is of order $m_{T} \simeq \sqrt{y^{2} f^{2}+M^{\prime 2}}$. Integrating out the top sector we find,

$$
\frac{c_{g}}{c_{g, \mathrm{SM}}}=\frac{c_{\gamma}}{c_{\gamma, \mathrm{SM}}}=\cos (\hat{v} / \sqrt{2} f)=\sqrt{1-\frac{v^{2}}{2 f^{2}}} .
$$

Thus, the top sector contribution to the Higgs dimension-5 interactions is reduced by a factor that is independent of the details of the top sector, such as the masses and the coupling of the top eigenstates. The interaction terms in eq. (5.9) arise e.g. in the Simplest Little Higgs model with an $[\mathrm{SU}(3) / \mathrm{SU}(2)]^{2}$ coset structure [60-65] when taking the limit $f_{2} \gg f_{1}$. In that case one also finds $c_{V}=c_{b}=\sqrt{1-\frac{v^{2}}{2 f^{2}}}$. Therefore, in the Simplest Little Higgs model, the rates in all Higgs channels are universally suppressed by a factor depending only on the compositeness scale: $\sigma / \sigma_{S M}=1-v^{2} / 2 f^{2}$. The same holds for the $\mathrm{SO}(5) / \mathrm{SO}(4)$ minimal composite Higgs with fermions embedded in the spinorial representation of $\mathrm{SO}(5)[66,67]$. Note that the independence of the Higgs widths of the fine details of the top sector persists in numerous Little Higgs and composite Higgs models [68-70], although it may not hold in more complicated models where the top couples to more than one composite operator [71].

Repeating the analysis done in previous sections, in figure 6 we present the constraints on $\xi \equiv v^{2} / f^{2}$ from the current LHC and Tevatron Higgs measurements. We find that, assuming a $125 \mathrm{GeV}$ Higgs boson, $\xi>1$ is excluded at the $95 \%$ CL. Note that as discussed above, all relative rates have similar dependence on $\xi$ and are therefore drawn on top of one another.

\subsection{Non-universal suppression}

Another phenomenologically distinct example with one top partner arises within the Twin Higgs scenario [72], where the global symmetry giving rise to a pseudo-Goldstone Higgs arises accidentally as a consequence of a discrete symmetry. In particular, in the left-right symmetric Twin Higgs model [73] the top sector interactions with the Higgs take the form

$$
-\mathcal{L}_{\text {top }}=y \sin (|H| / f) t T^{c}+y \cos (|H| / f) T t^{c}+M_{2} T T_{c} .
$$

Using the same methods as before one finds,

$$
c_{V}=c_{b}=\sqrt{1-\frac{v^{2}}{2 f^{2}}}, \quad \frac{c_{g}}{c_{g, \mathrm{SM}}}=\frac{c_{\gamma}}{c_{\gamma, \mathrm{SM}}}=\frac{1-\frac{v^{2}}{f^{2}}}{\sqrt{1-\frac{v^{2}}{2 f^{2}}}} .
$$

In this example, the Higgs partial width into gluons is modified by a different factor than that into $\mathrm{W}$ and $\mathrm{Z}$ bosons. The constraints on the non-universal suppression models are presented in figure 7. Assuming a 125 Gev Higgs, the allowed region at 95\% CL is $\xi<0.6$. 

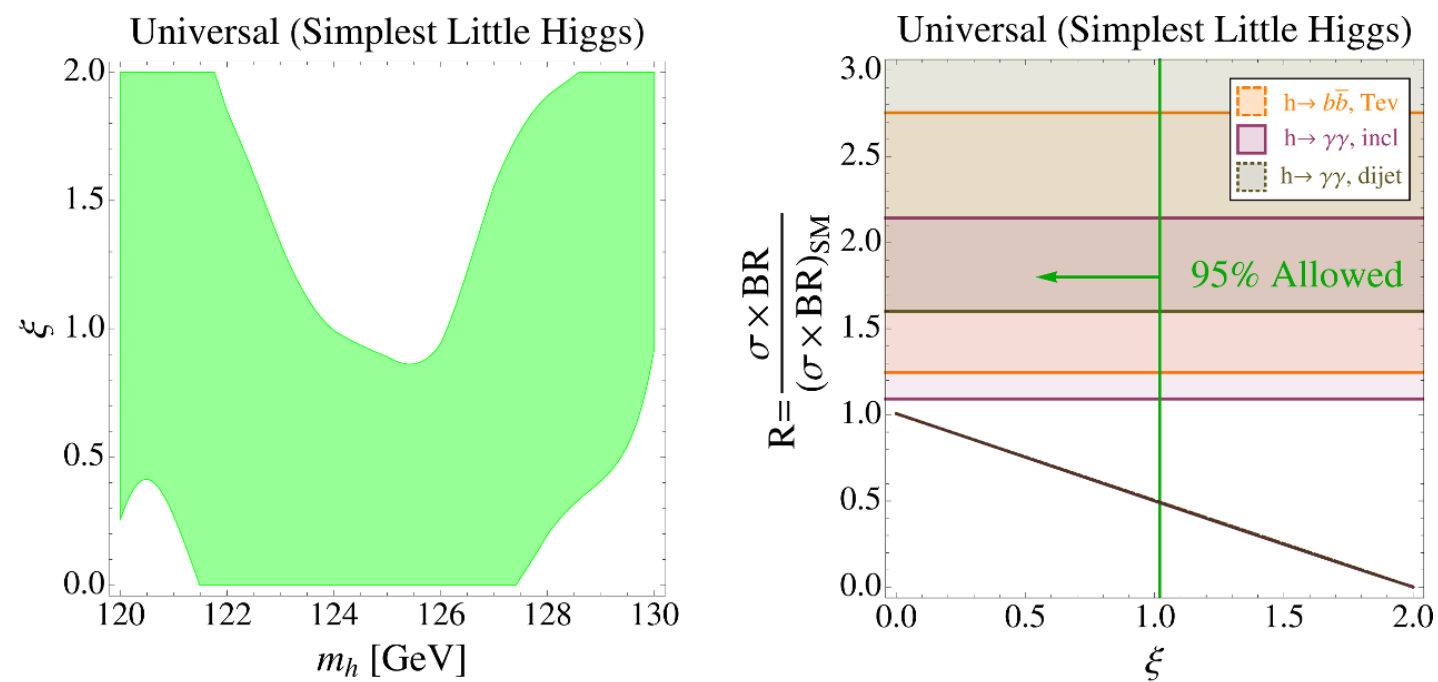

Figure 6. Left: Favored region, $95 \% \mathrm{CL}$, in the $\xi-m_{h}$ plane where $\xi \equiv \frac{v^{2}}{f^{2}}$, derived from the combination of all search channels, for models with universal suppression such as the Simplest Little Higgs model described in section 5.2. Right: Constraints for $m_{h}=125 \mathrm{GeV}$. The three bands show the $1 \sigma$ allowed regions: $R_{\gamma \gamma}$ (purple), $R_{b \bar{b}}$ (orange), $R_{\gamma \gamma j j}$ (beige). The three curves show the theoretical predictions as a function of $\xi: R_{\gamma \gamma}$ (solid-purple), $R_{b \bar{b}}$ (dashed-orange) and $R_{\gamma \gamma j j}$ (dotted-beige). Only 3 channels are shown, but all channels are included. Due to the universal suppression all three curves share the same dependence on $\xi$ and are therefore on top of one another. The region to the left of the green line at $\xi \simeq 1$ shows the $95 \%$ CL experimental (combined) allowed region.
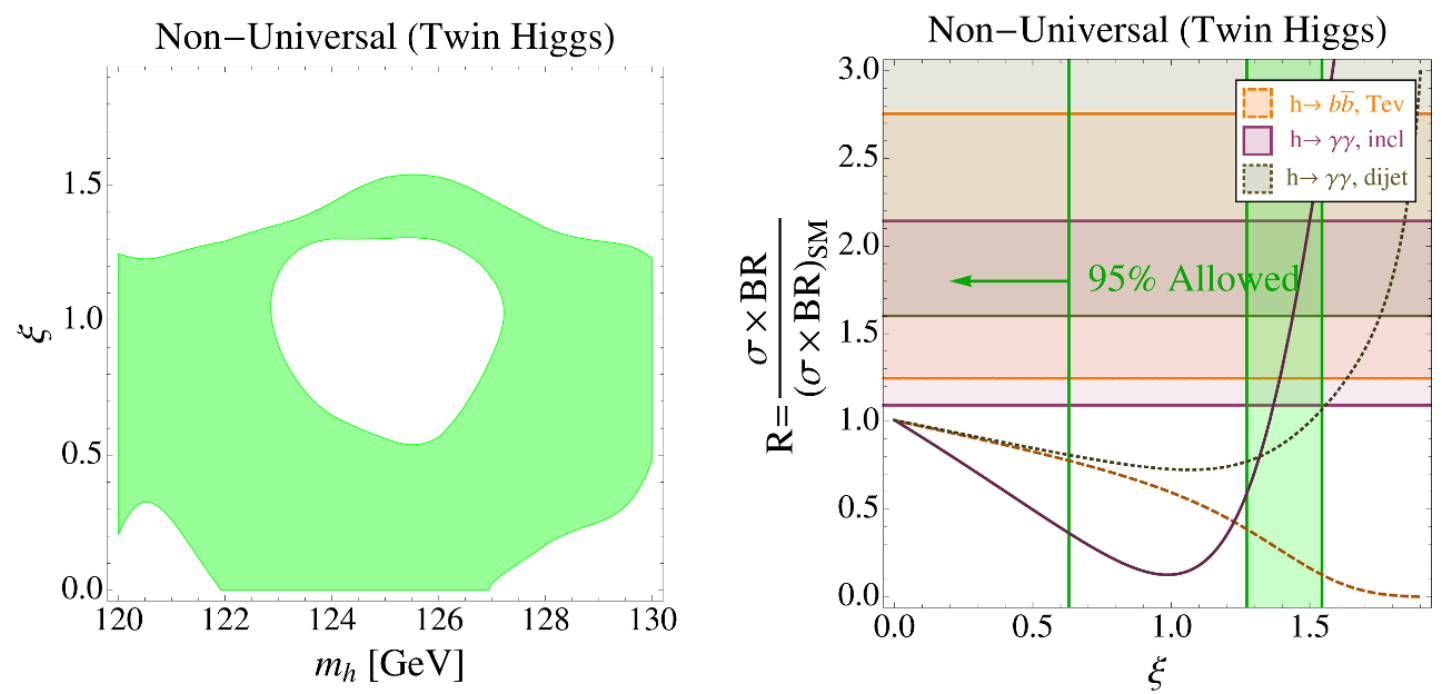

Figure 7. Left: Favored region, $95 \% \mathrm{CL}$, in the $\xi \equiv \frac{v^{2}}{f^{2}}-m_{h}$ plane, derived from the combination of all search channels, for the Twin Higgs model described in section 5.3. Right: Constraints for $m_{h}=125 \mathrm{GeV}$. The three bands show the $1 \sigma$ allowed regions: $R_{\gamma \gamma}$ (purple), $R_{b \bar{b}}$ (orange), $R_{\gamma \gamma j j}$ (beige). The three curves show the theoretical predictions as a function of $\xi: R_{\gamma \gamma}$ (solid-purple), $R_{b \bar{b}}$ (dashed-orange) and $R_{\gamma \gamma j j}$ (dotted-beige). Only 3 channels are shown, but all channels are included. The region to the left of the green line at $\xi \simeq 0.6$ shows the $95 \%$ CL experimental (combined) allowed region. 

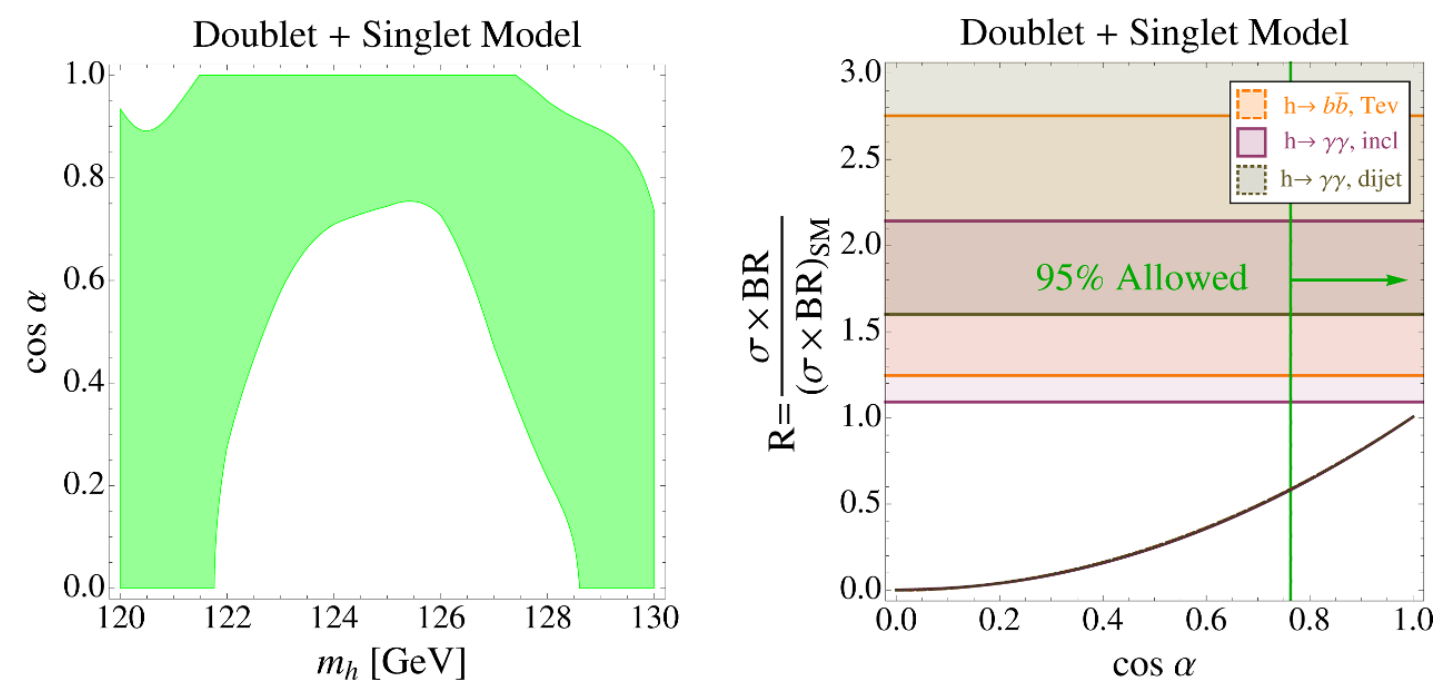

Figure 8. Left: Favored region, 95\% CL, in the $\cos \alpha-m_{h}$ plane, derived from the combination of all search channels, for the doublet-singlet model described in section 6.1. Right: Constraints for $m_{h}=125 \mathrm{GeV}$. The three bands show the $1 \sigma$ allowed regions: $R_{\gamma \gamma}$ (purple), $R_{b \bar{b}}$ (orange), $R_{\gamma \gamma j j}$ (beige). The three curves show the theoretical predictions as a function of $\cos \alpha: R_{\gamma \gamma}$ (solid-purple), $R_{b \bar{b}}$ (dashed-orange) and $R_{\gamma \gamma j j}$ (dotted-beige). Only 3 channels are shown, but all channels are included. The region to the right of the green line at $\cos \alpha \simeq 0.75$ shows the $95 \% \mathrm{CL}$ experimental (combined) allowed region.

\section{Multi-Higgs models}

\subsection{Doublet + singlet}

The simplest set-up with multiple Higgs bosons is the one with an electroweak-singlet scalar field mixing with the Higgs. As a result, the mass eigenstates are linear combinations of the Higgs scalar originating from the doublet (which couples to the SM matter) and singlet (which does not couple to matter). Denoting the mixing angle as $\alpha$, all the couplings of the Higgs boson are suppressed by $\cos \alpha$,

$$
c_{V}=c_{b}=\frac{c_{g}}{c_{g, \mathrm{SM}}}=\frac{c_{\gamma}}{c_{\gamma, \mathrm{SM}}}=\cos \alpha .
$$

As a consequence, the Higgs production and decay rates in all the channels are universally suppressed by $\cos ^{2} \alpha$. This is analogous to what happens in a fermionic model in section 5.2. The new element is the appearance of the second Higgs eigenstate, denoted by $H^{0}$, whose couplings are suppressed by $\sin \alpha$ compared to those of the SM Higgs boson, and whose mass is in general a free parameter. In figure 8 we present the LHC and Tevatron Higgs constraints on this model. We find rather strong constraints on the mixing of the doublet with the singlet, $\cos \alpha \gtrsim 0.70$. In deriving these constraints we assumed that $m_{H^{0}}>m_{h} / 2$.

\subsection{Two Higgs doublets}

We end with the study of 2 Higgs doublets $H_{u}, H_{d}$, the former coupling to up-type quarks, and the latter to down-type quarks and leptons. The physical fields are embedded into the 


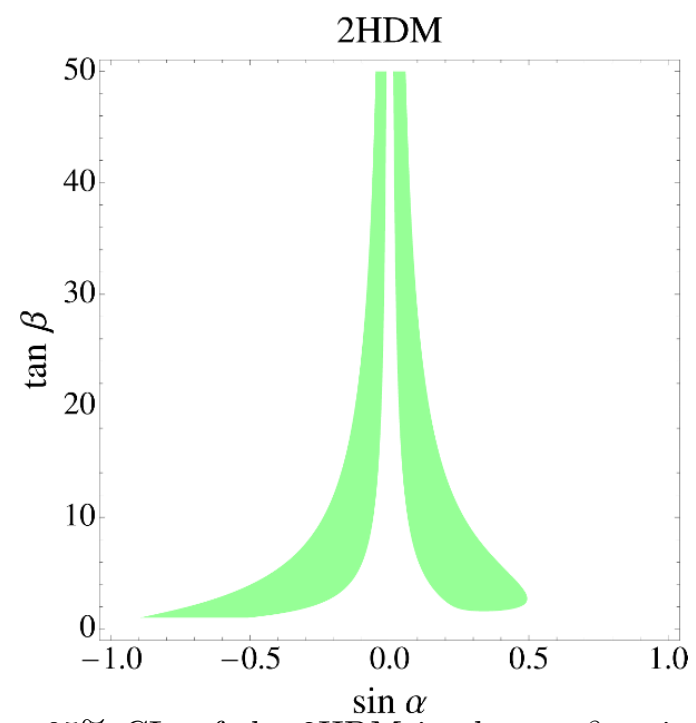

Figure 9. Favored region, 95\% CL, of the $2 \mathrm{HDM}$ in $\operatorname{the} \tan \beta-\sin \alpha$ plane, derived from the combination of all search channels. We take $m_{H^{ \pm}}=200 \mathrm{GeV}$, but a lighter charged Higgs would only slightly change the favored region. The favored region for $\sin \alpha<0$ concentrates around the decoupling limit, $\alpha=\beta-\pi / 2$, where all couplings are SM-like, whereas the region for $\sin \alpha>0$ lies around the region $\alpha=-\beta+\pi / 2$ where the top Yukawa coupling is SM-like.

doublets as,

$$
\begin{aligned}
& H_{u}=\left(\begin{array}{c}
\cos \beta H^{+} \\
\frac{1}{\sqrt{2}}\left(v \sin \beta+h \cos \alpha+H^{0} \sin \alpha+i A^{0} \cos \beta\right)
\end{array}\right), \\
& H_{d}=\left(\begin{array}{c}
\frac{1}{\sqrt{2}}\left(v \cos \beta-h \sin \alpha+H^{0} \cos \alpha+i A^{0} \sin \beta\right) \\
\sin \beta H^{-}
\end{array}\right) .
\end{aligned}
$$

The couplings of the lightest Higgs boson $h$ are described by two angle $\alpha, \beta$ who are in general free parameters. ${ }^{4}$ We find,

$$
c_{V}=\sin (\beta-\alpha), \quad c_{b}=-\frac{\sin \alpha}{\cos \beta}, \quad \frac{c_{g}}{c_{g, \mathrm{SM}}}=\frac{c_{\gamma}}{c_{\gamma, \mathrm{SM}}}=\frac{\cos \alpha}{\sin \beta} .
$$

By convention $0<\beta<\pi / 2$. In general, there is an additional contribution to $c_{\gamma}$ from the charged Higgs, but it is always small compared to the contribution from the W-boson.

The 2 Higgs Doublet Model $(2 \mathrm{HDM})$ can change all couplings to the Higgs and thus is highly constrained by the LHC Higgs searches [29-32, 74, 75]. In figure 9 we show the constraints in the $\tan \beta-\sin \alpha$ plane for $m_{H^{ \pm}}=200 \mathrm{GeV}$. Lighter masses would only slightly change the favored region. The favored region for $\sin \alpha<0$ concentrates around the decoupling limit $\alpha=\beta-\pi / 2$, where all couplings are SM-like. The region for $\sin \alpha>0$ lies around the region $\alpha=-\beta+\pi / 2$ where the top Yukawa coupling is SM-like.

\footnotetext{
${ }^{4}$ If the Higgs potential is that of the MSSM, the angle $\alpha$ is not independent of $m_{A^{0}}$ and $m_{H^{ \pm}}$. Furthermore, in that case $-\pi / 2<\alpha<0$ for $m_{A^{0}}>m_{Z}$.
} 


\section{Conclusions}

The indications for the existence of a Higgs boson provided recently by LHC and Tevatron are preliminary and may go away with more data. With this caveat in mind, it is interesting to ask whether the available experimental information is compatible with the SM Higgs boson, and whether it favors or disfavors any particular constructions beyond the SM. In this paper we analyzed recent LHC and Tevatron searches sensitive to a light (115-130 GeV) Higgs boson, combining results in the channels: $h \rightarrow \gamma \gamma$ (both gluon fusion and vectorboson fusion), $h \rightarrow Z Z^{*} \rightarrow 4 l, h \rightarrow W W^{*} \rightarrow 2 l 2 \nu$, and $h \rightarrow b \bar{b}$, as well as combining the LHC and Tevatron data. We presented interpretations of that combination in the context of several effective models, with the special emphasis on models addressing the naturalness problem of electroweak symmetry breaking.

We have argued that, unsurprisingly, the combination of the LHC and Tevatron data favors the Higgs boson in the mass range $124-126 \mathrm{GeV}$, with the best fit cross section close to the one predicted by the SM. Less trivially, we recast the LHC and Tevatron Higgs results as constraints on the parameters of the effective lagrangian at the scale $\sim m_{h}$ describing the leading interactions of the Higgs boson with the SM fields. Furthermore, we found that the data already put interesting constraints on simple natural new physics models, especially on those predicting suppression of $\sigma(p p \rightarrow h) \operatorname{Br}(h \rightarrow \gamma \gamma)$ and $\sigma(p p \rightarrow h j j) \operatorname{Br}(h \rightarrow \gamma \gamma)$. For example, in a model with one fermionic top partner stabilizing the Higgs potential, the top partner masses below $\sim 220 \mathrm{GeV}$ are disfavored at $95 \% \mathrm{CL}$. For one scalar partner the corresponding bound is $\sim 240 \mathrm{GeV}$, due to the fact that a single scalar stabilizing the Higgs potential always provides a positive contribution to $\Gamma(h \rightarrow \gamma \gamma)$. These bounds can be further relaxed for more complicated models. In particular in a model with 2 scalar partners the total contribution to $\Gamma(h \rightarrow \gamma \gamma)$ can be negligible even for very light scalars, at the expense of fine tuning.

We anticipate these bounds to significantly improve with additional data to be collected in 2012. Alternatively, studying the effective theory of the Higgs bosons may prove to be the shortest way to a discovery of new physics beyond the SM.

Note added. Right after our paper appeared, refs. [76] and [77] also appeared. The references also interpret the LHC Higgs results as constraints on the effective theory of Higgs interactions and overlap in part with our work. In order to assess compatibility with our results, in v2 we added the top right plot of figure 2, which can be directly compared to the contours in the $a-c$ plane presented in [76, 77]. In spite of using different statistical methods, we find very similar preferred regions in the $a-c$ plane. Nevertheless, our constraints on $\xi=v^{2} / f^{2}$ in section 5.2 are somewhat stronger than in [76]. We further note that our definition of $\xi$ differs by a factor of 2 compared to the definition in $[76,77]$.

\section{Acknowledgments}

We especially thank Patrick Meade for collaboration in the early stages of this project and for many useful discussions. We also thank David Curtin, Aielet Efrati, Yonit Hochberg, Yossi Nir and Gilad Perez for useful discussions. The work of DC, EK and TV is supported 
in part by a grant from the Israel Science Foundation. The work of TV is further supported in part by the US-Israel Binational Science Foundation and the EU-FP7 Marie Curie, CIG fellowship.

Open Access. This article is distributed under the terms of the Creative Commons Attribution License which permits any use, distribution and reproduction in any medium, provided the original author(s) and source are credited.

\section{References}

[1] ATLAS collaboration, G. Aad et al., Combined search for the Standard Model Higgs boson using up to $4.9 \mathrm{fb}^{-1}$ of pp collision data at $\sqrt{s}=7 \mathrm{TeV}$ with the ATLAS detector at the LHC, Phys. Lett. B 710 (2012) 49 [arXiv:1202.1408] [INSPIRE].

[2] CMS collaboration, S. Chatrchyan et al., Combined results of searches for the standard model Higgs boson in pp collisions at $\sqrt{s}=7$ TeV, Phys. Lett. B 710 (2012) 26 [arXiv: 1202.1488] [INSPIRE].

[3] CMS collaboration, Combination of SM, SM4, FP Higgs boson searches, PAS-HIG-12-008

[4] ATLAS collaboration, An update to the combined search for the Standard Model Higgs boson with the ATLAS detector at the LHC using up to $4.9 \mathrm{fb}^{-1}$ of pp collision data at $\sqrt{s}=7 \mathrm{TeV}$, ATLAS-CONF-2012-019 (2012).

[5] ATLAS collaboration, G. Aad et al., Search for the Standard Model Higgs boson in the diphoton decay channel with $4.9 \mathrm{fb}^{-1}$ of pp collisions at $\sqrt{\mathrm{s}}=7 \mathrm{TeV}$ with ATLAS, Phys. Rev. Lett. 108 (2012) 111803 [arXiv:1202.1414] [INSPIRE].

[6] ATLAS collaboration, G. Aad et al., Search for the Standard Model Higgs boson in the decay channel $H \rightarrow Z Z^{(*)} \rightarrow 4 l$ with $4.8 \mathrm{fb}^{-1}$ of pp collision data at $\sqrt{s}=7 \mathrm{TeV}$ with ATLAS, Phys. Lett. B 710 (2012) 383 [arXiv:1202.1415] [InSPIRE].

[7] CMS collaboration, M. Pieri, Searches for the Standard Model Higgs Boson at CMS, arXiv:1205.2907 [INSPIRE].

[8] CMS collaboration, S. Chatrchyan et al., Search for the standard model Higgs boson decaying into two photons in pp collisions at $\sqrt{s}=7 \mathrm{TeV}$, Phys. Lett. B 710 (2012) 403 [arXiv: 1202.1487] [INSPIRE].

[9] CMS collaboration, A search using multivariate techniques for a standard model Higgs boson decaying into two photons, PAS-HIG-12-001.

[10] CMS collaboration, S. Chatrchyan et al., Search for the standard model Higgs boson in the decay channel $H \rightarrow Z Z \rightarrow 4$ leptons in pp collisions at $\sqrt{s}=7$ TeV, Phys. Rev. Lett. 108, 111804 (2012) [arXiv:1202.1997] [INSPIRE].

[11] CMS collaboration, S. Chatrchyan et al., Search for the standard model Higgs boson decaying to a W pair in the fully leptonic final state in pp collisions at $\sqrt{s}=7 \mathrm{TeV}$, Phys. Lett. B 710 (2012) 91 [arXiv:1202.1489] [INSPIRE].

[12] CMS collaboration, S. Chatrchyan et al., Search for a Higgs boson in the decay channel $H \rightarrow Z Z^{(*)} \rightarrow q \bar{q} l^{-} l^{+}$in pp collisions at $\sqrt{s}=7 \mathrm{TeV}$, JHEP 04 (2012) 036 [arXiv: 1202.1416] [INSPIRE].

[13] ATLAS collaboration, Search for the Standard Model Higgs boson in the $H \rightarrow W W \rightarrow l \nu l \nu$ decay mode with $4.7 \mathrm{fb}^{-1}$ of ATLAS data at $\sqrt{s}=7 \mathrm{TeV}$, ATLAS-CONF-2012-012 (2012). 
[14] Tevnph (Tevatron New Phenomina and Higgs Working Group), CDF, D0 collaborations, Combined CDF and DO Search for Standard Model Higgs Boson Production with up to $10.0 \mathrm{fb}^{-1}$ of Data, arXiv:1203.3774 [INSPIRE].

[15] ATLAS collaboration, G. Aad et al., Search for the Standard Model Higgs boson in the

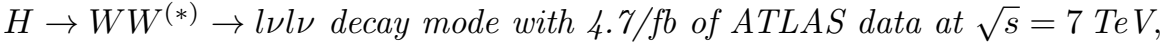
arXiv: 1206.0756 [INSPIRE].

[16] J. Espinosa, C. Grojean and M. Muhlleitner, Composite Higgs under LHC Experimental Scrutiny, EPJ Web Conf. 28 (2012) 08004 [arXiv:1202.1286] [INSPIRE].

[17] L.J. Hall, D. Pinner and J.T. Ruderman, A Natural SUSY Higgs Near 126 GeV, JHEP 04 (2012) 131 [arXiv:1112.2703] [inSPIRE].

[18] A. Arbey, M. Battaglia, A. Djouadi, F. Mahmoudi and J. Quevillon, Implications of a 125 GeV Higgs for supersymmetric models, Phys. Lett. B 708 (2012) 162 [arXiv:1112.3028] [INSPIRE].

[19] S. Heinemeyer, O. Stal and G. Weiglein, Interpreting the LHC Higgs Search Results in the MSSM, Phys. Lett. B 710 (2012) 201 [arXiv:1112.3026] [inSPIRE].

[20] P. Draper, P. Meade, M. Reece and D. Shih, Implications of a 125 GeV Higgs for the MSSM and Low-Scale SUSY Breaking, Phys. Rev. D 85 (2012) 095007 [arXiv:1112.3068] [INSPIRE].

[21] M. Carena, S. Gori, N.R. Shah and C.E. Wagner, A 125 GeV SM-like Higgs in the MSSM and the $\gamma \gamma$ rate, JHEP 03 (2012) 014 [arXiv:1112.3336] [INSPIRE].

[22] U. Ellwanger, A Higgs boson near $125 \mathrm{GeV}$ with enhanced di-photon signal in the NMSSM, JHEP 03 (2012) 044 [arXiv: 1112.3548] [INSPIRE].

[23] M. Kadastik, K. Kannike, A. Racioppi and M. Raidal, Implications of the $125 \mathrm{GeV}$ Higgs boson for scalar dark matter and for the CMSSM phenomenology, JHEP 05 (2012) 061 [arXiv: 1112.3647] [INSPIRE].

[24] J. Cao, Z. Heng, D. Li and J.M. Yang, Current experimental constraints on the lightest Higgs boson mass in the constrained MSSM, Phys. Lett. B 710 (2012) 665 [arXiv:1112.4391] [INSPIRE].

[25] A. Arvanitaki and G. Villadoro, A Non Standard Model Higgs at the LHC as a Sign of Naturalness, JHEP 02 (2012) 144 [arXiv:1112.4835] [INSPIRE].

[26] J.F. Gunion, Y. Jiang and S. Kraml, The Constrained NMSSM and Higgs near $125 \mathrm{GeV}$, Phys. Lett. B 710 (2012) 454 [arXiv:1201.0982] [INSPIRE].

[27] S. King, M. Muhlleitner and R. Nevzorov, NMSSM Higgs Benchmarks Near 125 GeV, Nucl. Phys. B 860 (2012) 207 [arXiv: 1201.2671] [INSPIRE].

[28] Z. Kang, J. Li and T. Li, On Naturalness of the (N)MSSM, arXiv:1201.5305 [INSPIRE].

[29] P. Ferreira, R. Santos, M. Sher and J.P. Silva, Implications of the LHC two-photon signal for two-Higgs-doublet models, arXiv:1112.3277 [INSPIRE].

[30] P. Ferreira, R. Santos, M. Sher and J.P. Silva, Could the LHC two-photon signal correspond to the heavier scalar in two-Higgs-doublet models?, Phys. Rev. D 85 (2012) 035020 [arXiv: 1201.0019] [INSPIRE]. 
[31] E. Cervero and J.-M. Gerard, Minimal violation of flavour and custodial symmetries in a vectophobic Two-Higgs-Doublet-Model, Phys. Lett. B 712 (2012) 255 [arXiv:1202.1973] [INSPIRE].

[32] K. Blum and R.T. D'Agnolo, 2 Higgs or not 2 Higgs, arXiv:1202.2364 [INSPIRE].

[33] C. Englert, T. Plehn, M. Rauch, D. Zerwas and P.M. Zerwas, LHC: Standard Higgs and Hidden Higgs, Phys. Lett. B 707 (2012) 512 [arXiv:1112.3007] [INSPIRE].

[34] C. Cheung and Y. Nomura, Higgs Descendants, arXiv:1112.3043 [INSPIRE].

[35] G. Guo, B. Ren and X.-G. He, LHC Evidence Of A 126 GeV Higgs Boson From $H \rightarrow \gamma \gamma$ With Three And Four Generations, arXiv:1112.3188 [INSPIRE].

[36] A. Djouadi, O. Lebedev, Y. Mambrini and J. Quevillon, Implications of LHC searches for Higgs-portal dark matter, Phys. Lett. B 709 (2012) 65 [arXiv:1112.3299] [INSPIRE].

[37] K. Cheung and T.-C. Yuan, Could the excess seen at 124-126 GeV be due to the Randall-Sundrum Radion?, Phys. Rev. Lett. 108 (2012) 141602 [arXiv:1112.4146] [INSPIRE].

[38] B. Batell, S. Gori and L.-T. Wang, Exploring the Higgs Portal with 10/fb at the LHC, JHEP 06 (2012) 172 [arXiv: 1112.5180] [INSPIRE].

[39] J.F. Kamenik and C. Smith, Could a light Higgs boson illuminate the dark sector?, Phys. Rev. D 85 (2012) 093017 [arXiv:1201.4814] [INSPIRE].

[40] A. Falkowski, S. Rychkov and A. Urbano, What if the Higgs couplings to $W$ and $Z$ bosons are larger than in the Standard Model?, JHEP 04 (2012) 073 [arXiv:1202.1532] [INSPIRE].

[41] E. Gabrielli, B. Mele and M. Raidal, Has a fermiophobic Higgs boson been detected at the $L H C$ ?, arXiv:1202.1796 [INSPIRE].

[42] F. Bonnet, M. Gavela, T. Ota and W. Winter, Anomalous Higgs couplings at the LHC and their theoretical interpretation, Phys. Rev. D 85 (2012) 035016 [arXiv:1105.5140] [INSPIRE].

[43] C. Englert, T. Plehn, D. Zerwas and P.M. Zerwas, Exploring the Higgs portal, Phys. Lett. B 703 (2011) 298 [arXiv: 1106.3097] [INSPIRE].

[44] C. Englert, J. Jaeckel, E. Re and M. Spannowsky, Evasive Higgs Maneuvers at the LHC, Phys. Rev. D 85 (2012) 035008 [arXiv:1111.1719] [INSPIRE].

[45] B.A. Dobrescu, G.D. Kribs and A. Martin, Higgs Underproduction at the LHC, Phys. Rev. D 85 (2012) 074031 [arXiv: 1112.2208] [InSPIRE].

[46] A. Drozd, B. Grzadkowski and J. Wudka, Multi-Scalar-Singlet Extension of the Standard Model - the Case for Dark Matter and an Invisible Higgs Boson, JHEP 04 (2012) 006 [arXiv: 1112.2582] [INSPIRE].

[47] A. Djouadi, The Anatomy of electro-weak symmetry breaking. I: The Higgs boson in the standard model, Phys. Rept. 457 (2008) 1 [hep-ph/0503172] [INSPIRE].

[48] A. Djouadi, The Anatomy of electro-weak symmetry breaking. II: The Higgs bosons in the minimal supersymmetric model, Phys. Rept. 459 (2008) 1 [hep-ph/0503173] [INSPIRE].

[49] J.R. Ellis, M.K. Gaillard and D.V. Nanopoulos, A Phenomenological Profile of the Higgs Boson, Nucl. Phys. B 106 (1976) 292 [InSPIRE].

[50] M.A. Shifman, A. Vainshtein, M. Voloshin and V.I. Zakharov, Low-Energy Theorems for Higgs Boson Couplings to Photons, Sov. J. Nucl. Phys. 30 (1979) 711 [InSPIRE]. 
[51] J. Alwall, M. Herquet, F. Maltoni, O. Mattelaer and T. Stelzer, MadGraph 5: Going Beyond, JHEP 06 (2011) 128 [arXiv: 1106.0522] [INSPIRE].

[52] S. Ovyn, X. Rouby and V. Lemaitre, DELPHES, a framework for fast simulation of a generic collider experiment, arXiv:0903.2225 [INSPIRE].

[53] R. Dermisek and I. Low, Probing the Stop Sector and the Sanity of the MSSM with the Higgs Boson at the LHC, Phys. Rev. D 77 (2008) 035012 [hep-ph/0701235] [INSPIRE].

[54] M. Carena, G. Nardini, M. Quirós and C. Wagner, The Baryogenesis Window in the MSSM, Nucl. Phys. B 812 (2009) 243 [arXiv:0809.3760] [inSPIRE].

[55] A. Menon and D.E. Morrissey, Higgs Boson Signatures of MSSM Electroweak Baryogenesis, Phys. Rev. D 79 (2009) 115020 [arXiv: 0903.3038] [InSPIRE].

[56] T. Cohen and A. Pierce, Electroweak Baryogenesis and Colored Scalars, Phys. Rev. D 85 (2012) 033006 [arXiv:1110.0482] [INSPIRE].

[57] H.-C. Cheng and I. Low, TeV symmetry and the little hierarchy problem, JHEP 09 (2003) 051 [hep-ph/0308199] [INSPIRE].

[58] H.-C. Cheng, I. Low and L.-T. Wang, Top partners in little Higgs theories with T-parity, Phys. Rev. D 74 (2006) 055001 [hep-ph/0510225] [INSPIRE].

[59] I. Low, R. Rattazzi and A. Vichi, Theoretical Constraints on the Higgs Effective Couplings, JHEP 04 (2010) 126 [arXiv:0907.5413] [INSPIRE].

[60] M. Schmaltz, The Simplest little Higgs, JHEP 08 (2004) 056 [hep-ph/0407143] [INSPIRE].

[61] D.E. Kaplan and M. Schmaltz, The Little Higgs from a simple group, JHEP 10 (2003) 039 [hep-ph/0302049] [INSPIRE].

[62] M. Schmaltz and D. Tucker-Smith, Little Higgs review, Ann. Rev. Nucl. Part. Sci. 55 (2005) 229 [hep-ph/0502182] [inSPIRE].

[63] M. Perelstein, Little Higgs models and their phenomenology, Prog. Part. Nucl. Phys. 58 (2007) 247 [hep-ph/0512128] [inSPIRE].

[64] T. Han, H.E. Logan, B. McElrath and L.-T. Wang, Phenomenology of the little Higgs model, Phys. Rev. D 67 (2003) 095004 [hep-ph/0301040] [InSPIRE].

[65] T. Han, H.E. Logan, B. McElrath and L.-T. Wang, Loop induced decays of the little Higgs: $H \rightarrow g g, \gamma \gamma$, Phys. Lett. B 563 (2003) 191 [Erratum ibid. B 603 (2004) 257] [hep-ph/0302188] [INSPIRE].

[66] K. Agashe, R. Contino and A. Pomarol, The Minimal composite Higgs model, Nucl. Phys. B 719 (2005) 165 [hep-ph/0412089] [INSPIRE].

[67] J. Espinosa, C. Grojean and M. Muhlleitner, Composite Higgs Search at the LHC, JHEP 05 (2010) 065 [arXiv: 1003.3251] [InSPIRE].

[68] A. Falkowski, Pseudo-goldstone Higgs production via gluon fusion, Phys. Rev. D 77 (2008) 055018 [arXiv: 0711.0828] [INSPIRE].

[69] G. Cacciapaglia, A. Deandrea and J. Llodra-Perez, $H \rightarrow \gamma \gamma$ beyond the Standard Model, JHEP 06 (2009) 054 [arXiv: 0901.0927] [INSPIRE].

[70] I. Low and A. Vichi, On the production of a composite Higgs boson, Phys. Rev. D 84 (2011) 045019 [arXiv: 1010 . 2753] [INSPIRE]. 
[71] A. Azatov and J. Galloway, Light Custodians and Higgs Physics in Composite Models, Phys. Rev. D 85 (2012) 055013 [arXiv:1110.5646] [INSPIRE].

[72] Z. Chacko, H.-S. Goh and R. Harnik, The Twin Higgs: Natural electroweak breaking from mirror symmetry, Phys. Rev. Lett. 96 (2006) 231802 [hep-ph/0506256] [INSPIRE].

[73] Z. Chacko, H.-S. Goh and R. Harnik, A Twin Higgs model from left-right symmetry, JHEP 01 (2006) 108 [hep-ph/0512088] [INSPIRE].

[74] A. Akeroyd, Fermiophobic and other nonminimal neutral Higgs bosons at the LHC, J. Phys. G G 24 (1998) 1983 [hep-ph/9803324] [INSPIRE].

[75] B. Grzadkowski and P. Osland, Tempered Two-Higgs-Doublet Model, Phys. Rev. D 82 (2010) 125026 [arXiv: 0910 .4068] [INSPIRE].

[76] A. Azatov, R. Contino and J. Galloway, Model-Independent Bounds on a Light Higgs, JHEP 04 (2012) 127 [arXiv: 1202.3415] [INSPIRE].

[77] J. Espinosa, C. Grojean, M. Muhlleitner and M. Trott, Fingerprinting Higgs Suspects at the LHC, JHEP 05 (2012) 097 [arXiv: 1202.3697] [INSPIRE]. 